\title{
Interpregnancy intervals, birth outcomes and educational needs
}

\author{
Woods $\mathrm{NK}^{1 *}$, Schmidt CRA ${ }^{2}$, Reyes $\mathrm{J}^{3}$ and Engel $\mathrm{M}^{4}$ \\ ${ }^{1}$ Assistant Professor of Public Health at Wichita State University in Wichita, Kansas, USA \\ ${ }^{2}$ Associate Research Professor at University of Kansas School of Medicine-Wichita, Wichita, Kansas, USA \\ ${ }^{3}$ Department of Communication Sciences and Disorders, doctoral student at Wichita State University, Wichita, Kansas, USA \\ ${ }^{4}$ Research associate at University of Kansas School of Medicine-Wichita, Wichita, Kansas, USA
}

\begin{abstract}
Inter-Pregnancy Interval (IPI) is defined as the time between pregnancies; a short IPI has been associated with negative birth outcomes. The goal of this retrospective cohort study was to examine existing IPI data from all Kansas women in the United States from 2005-2013 who had more than one live singleton birth. Unmatched and matched births were used to model the odds of preterm birth, small for gestational age birth, and low birth weight with IPI as the predictor variable of interest. The data set included 25,754 singleton births. The incidence rate of preterm birth for second births was 7.0\%, small for gestational age was $8.6 \%$, and 5.0\% for low birth weight; third births rates were $7.1 \%, 8.2 \%$ and $5.0 \%$, respectively. Short IPIs may not be causally associated with low birthweight, preterm birth, and small for gestational age. However short IPIs should still be used as an indicator of risk status.
\end{abstract}

\section{Introduction}

Inter-pregnancy interval (IPI) is defined as the time between pregnancies. It is theorized that a short IPI does not allow the mother to replenish her reserves of necessary nutrients (protein) and micronutrients (iron and folic acid) to support fetal growth [1]. In this environment of severely low resources the body often gives preference to the mother [1]. The relationship between IPI and morbidity and mortality has been well studied [2]. While there is no standard definition for a "short" IPI, those less than 18 months have been linked to the following adverse outcomes: increased odds of preterm birth, a Small for Gestational Age (SGA) infant, congenital malformations, and infant mortality [3-8]. IPIs less than 6 months have been related to increased risk of low birth weight and adverse maternal outcomes, including 3rd trimester bleeding, premature rupture of membranes, anemia, puerperal endometriosis and death $[2,3]$.

Children born following a short IPI were reported to be at an increased risk for behavior problems and lower cognitive function by age six [9]. In addition, these children were at increased risk of neglect and exposure to maltreatment $[9,10]$. Mothers at greatest risk for short IPI include mothers who entered prenatal care after the first trimester and those who received inadequate prenatal care [11]. These relationships were most pronounced for women with less than a college degree. Postnatal care may also be a factor as a retrospective study of California's Medicaid database found mothers who did not receive contraception at a postpartum healthcare visit were more likely to have a short ( $<18$ month) IPI or very short ( $<6$ month) IPI when controlling for other predictor variables and covariates [12]. However, only $41 \%$ of mothers had a contraception claim within 90 days. Based on this study, six women would need to receive contraception to avoid one short IPI. In addition, long-acting reversible contraception (e.g. intrauterine devices) had higher odds of achieving IPIs of greater than
18 months compared to those on user-dependent hormonal methods (e.g. oral contraceptives) [13].

Despite recommendations against short IPI, a retrospective cohort study set in Australia, failed to find a significant relationship between short IPI and morbidities frequently reported in the literature [14]. Additional studies were recommended by authors to extend the generalizability of the findings. The goal of this study was to examine existing IPI data using both matched and unmatched analyses to inform future community-based IPI interventions and extend the generalizability of previous within mother analyses.

\section{Methods}

\section{Participants}

The retrospective data included all Kansas women who gave birth to three or more singleton infants between January 2005 and December 2013. Preterm birth, SGA, and low birth weight were the identified variables of interest to investigate associations related to IPI. Preterm birth was defined as a birth before the start of the $37^{\text {th }}$ week of gestation, SGA was defined as an infant birth weight below $10^{\text {th }}$ percentile for gestational age, and low birth weight was defined as weight of infant at delivery less than 2,500 grams [15]. The study protocol was reviewed and approved by a university Institutional Review Board (IRB).

Correspondence to: Nikki Keene Woods, Assistant Professor of Public Health at Wichita State University in Wichita, Kansas, USA, E-mail: Nikki.keenewoods@ wichita.edu

Key words: interpregnancy intervals, birth spacing, preterm birth, low birthweight, small for gestational age

Received: February 28, 2017; Accepted: March 18, 2017; Published: March 21, 2017 


\section{Study design and analysis}

Retrospective cohort study with Kansas vital statistics birth data from the state health department. Methods for analysis were comparable to Ball et al. (2014) [14]. Unmatched and matched births were used to model the odds of preterm birth, SGA birth, and low birth weight with IPI as the predictor variable of interest. IPIs were categorized for comparison to the reference interval of 18-23 months. IPI was categorized as follows: 0-5 months, 6-11 months, $12-17$ months, 18-23 months, 24-59 months, 60-108 months. Both the matched and unmatched models were adjusted for the potential confounding variables of maternal age, parity, and the use of Women, Infants, and Children (WIC) services. These confounds may vary both between and within mothers and are known to be related, directly or indirectly, to adverse birth outcomes. Maternal level of education has shown to be related to birth outcomes, but this variable is highly correlated with age and the use of WIC services ( $r>0.5)$ so it was not used in regression modeling to prevent issues of collinearity [16].

The maternally matched model used outcomes and associated interpregnancy interval preceding for the second and third recorded births of each woman within our data set. These represented the second and third live births recorded from each woman within our study time period and not necessarily each woman's second and third live birth. For example, a woman may have given birth to her third, fourth, and fifth child during our study period; however, the outcomes from the fourth and fifth births were used in our analysis. For this reason, our models were adjusted for parity. The SPSS COXREG command was used to run the conditional logistic regression analysis to identify the relationship between IPI and adverse birth outcomes within women. To identify the relationship between IPI and adverse birth outcomes between women, a logistic regression was run comparing the odds of preterm birth, small for gestational age birth, and low birth weight for the third births recorded in the data set. The SPSS GENLIN command was used to run the unconditional logistic regression in order to adjust the standard errors and confidence intervals based on the model deviance.

\section{Results}

The data set included 25,754 singleton births that were separated into 12,877 maternally matched pairs of births. Among second births, the incidence rate of preterm birth was $7.0 \%$, there were $8.6 \%$ small for gestational age, and 5.0\% for low birth weight. Among third births, the incidence rates of preterm birth, SGA and low birth weight were $7.1 \%, 8.2 \%$ and $5.0 \%$, respectively. A summary of birth outcomes by IPI, maternal race/ethnicity, maternal education, maternal age, and use of WIC services are shown in Table 1.

\section{Unmatched model}

For the unadjusted model, every interval except 12-17 months was associated with preterm birth when compared to the reference IPI interval. Odds ratios for preterm birth for a short IPI range from 1.14 to 2.54. For a long IPI the odds ratio for preterm birth ranged from 1.23 to 1.62 . These relationships, with the exception of a 12-17 month IPI, remained statistically significant after adjusting for maternal age, parity, and WIC services (Table 2). Small for gestational age births were statistically associated with an IPI of $0-5$ months (OR 1.30) when compared against the reference IPI; however, this was not statistically significant in the adjusted model (Table 2). Low birth weight was associated with an IPI of 0-5 months (OR 2.05) in comparison to the reference IPI. After adjusting for maternal age, parity and the use of
WIC services, an IPI of 0-5 months (1.39) had statistically significant increased odds of a low birth weight outcome.

\section{Matched model}

For matched models, the informative data come from birth pairs that have differing outcomes. Either the second birth or third birth resulted in one of the adverse outcomes of interest (preterm birth, small for gestational age birth, or low birth weight) while the other birth in the birth pair did not result in an adverse outcome. For low birth weight, small for gestational age birth, and preterm birth there were 951 (3.7\%), 1,542 (6.0\%), and 1,279 (5.0\%) informative pairs, respectively. For the unadjusted model, a short IPI ( $0-5$ months) was associated with preterm birth (OR 1.46). This relationship remained (OR 1.42) after adjusting for maternal age, parity, and WIC services (Table 2). When compared to an IPI of 18-23 months, no other IPI was statistically associated with an increase in adverse birth outcomes in the unadjusted models. A short IPI (0-5 months) was shown to have a slight protective relationship with regard to SGA birth (OR 0.78) in the adjusted model (Table 2).

The adjusted matched models were statistically significant for SGA $\left[\chi^{2}(8, N=3,669)=15.86, P=0.04\right]$ and preterm birth $\left[\chi^{2}(8, N=3,069)=\right.$ $16.21, P=0.04]$. The adjusted model for low birth weight did not reach statistical significance $\left[\chi^{2}(8, \mathrm{~N}=2,205)=13.24, P=0.10\right]$.

\section{Discussion}

This study provided important information regarding the analysis of IPI data. Similar to a previous analysis conducted by Ball et al. [14], the conditional approach accounted for each woman's overall risk of adverse birth outcomes among all of her children included in the analysis. Previous researchers have suggested this design removes the effects of measured or unmeasured maternal factors that are either fixed (such as genetic predisposition) or strongly correlated over time (such as long term health or other risk and protective factors across the lifespan) [17]. In effect, this analysis allowed for the examination of inferences that were based on mother effects. In comparison, the traditional approach of unconditional logistic regression is based on differences between women. The results from this study provide additional evidence to support the existence of unmeasured persistent maternal confounders.

Results also support that low birthweight, preterm birth, and SGA are not the result of short IPIs but are due to correlated maternal factors in the context of a developed country similar to Ball et al. [14]. These factors such as exposure to high-levels of stress for long periods of time, lifestyle factors or socioeconomic status are typically difficult to measure.

Future research should further explore IPIs to identify potential action points for changing 'pathways' based on risk and protective factors of correlated maternal factors for adverse birth outcomes. Using the Life Course Theory (LCT), a conceptual framework frequently used in the Maternal and Child Health field, can provide an overall analytic framework to guide study methods. LCT focuses on broad social, economic, and environmental factors as underlying causes of persistent inequalities in birth outcomes. This theory is focused and includes points from social determinants, social equity, and built environment models. LCT emphasizes several key concepts to improve birth outcomes and related infant mortality based on scientific evidence from reproductive health sciences and chronic disease research including: pathways/trajectories and risk/protective 
Table 1. Maternal characteristics for births used in analyses.

\begin{tabular}{|c|c|c|c|c|c|c|c|c|}
\hline & \multicolumn{4}{|c|}{ Second births } & \multicolumn{4}{|c|}{ Third births } \\
\hline & Total & Preterm (\%) & SGA(\%) & LBW (\%) & Total & Preterm (\%) & SGA(\%) & LBW (\%) \\
\hline & 12877 & $895(7.0)$ & $1105(8.6)$ & $641(5.0)$ & 12877 & $920(7.1)$ & $1056(8.2)$ & $639(5.0)$ \\
\hline \multicolumn{9}{|c|}{ Interpregnancy Interval (months) } \\
\hline $0-5$ & 1430 & $175(12.2)$ & $135(9.4)$ & $129(9.0)$ & 1172 & $151(12.9)$ & $118(10.1)$ & $102(8.7)$ \\
\hline 6-11 & 2893 & $188(6.5)$ & $272(9.4)$ & $140(4.8)$ & 2363 & $179(7.6)$ & $196(8.3)$ & $118(5.0)$ \\
\hline $12-17$ & 3158 & $197(6.2)$ & $214(7.6)$ & $129(4.1)$ & 2536 & $157(6.2)$ & $199(7.8)$ & $102(4.0)$ \\
\hline $18-23$ & 2256 & $118(5.2)$ & $172(7.6)$ & $83(3.7)$ & 2155 & $120(5.6)$ & $170(7.9)$ & $96(4.5)$ \\
\hline $24-59$ & 3029 & $210(6.9)$ & $271(8.9)$ & $155(5.1)$ & 4429 & $294(6.6)$ & $353(8.0)$ & $211(4.8)$ \\
\hline 60-119 & 111 & $7(6.3)$ & $14(12.6)$ & $5(4.5)$ & 222 & $19(8.6)$ & $20(9.0)$ & $10(4.5)$ \\
\hline \multicolumn{9}{|l|}{ Maternal Ethnicity ${ }^{\mathrm{a}}$} \\
\hline White-NH & 9748 & $585(6.0)$ & $727(7.5)$ & $402(4.1)$ & 9733 & $647(6.6)$ & $687(7.1)$ & $407(4.2)$ \\
\hline Black-NH & 1023 & $138(13.5)$ & $176(17.2)$ & $110(10.8)$ & 1018 & $123(12.1)$ & $158(15.5)$ & $122(12.0)$ \\
\hline Asian-NH & 201 & $15(7.5)$ & $19(9.5)$ & $12(6.0)$ & 202 & $10(5.0)$ & $29(14.4)$ & $8(4.0)$ \\
\hline Native Am-NH & 68 & $9(13.2)$ & $6(8.8)$ & $8(11.8)$ & 68 & $7(10.3)$ & $8(11.8)$ & $6(8.8)$ \\
\hline Nat Hawaiian OPI-NH & 8 & $1(12.5)$ & $3(37.5)$ & $1(12.5)$ & 12 & $1(8.3)$ & $2(16.7)$ & $1(8.3)$ \\
\hline Other-NH & 40 & $5(12.5)$ & $5(12.5)$ & $3(7.5)$ & 37 & $1(2.7)$ & $2(5.4)$ & $0(0.0)$ \\
\hline Multi-NH & 192 & $21(10.9)$ & $24(12.5)$ & $13(6.8)$ & 183 & $15(8.2)$ & $20(10.9)$ & $17(9.3)$ \\
\hline Unknown-NH & 5 & $0(0.0)$ & $1(20.0)$ & $0(0.0)$ & 5 & $1(20)$ & $0(0.0)$ & $1(20.0)$ \\
\hline Hispanic & 1592 & $121(7.6)$ & $144(9.0)$ & $92(5.8)$ & 1619 & $115(7.1)$ & $150(9.3)$ & $77(4.8)$ \\
\hline \multicolumn{9}{|l|}{ Maternal Education } \\
\hline$<9$ th Grade & 357 & $33(9.2)$ & $35(9.8)$ & $20(5.6)$ & 344 & $28(8.1)$ & $35(10.2)$ & $16(4.7)$ \\
\hline 9-12; No Diploma & 1909 & $192(10.1)$ & $252(13.2)$ & $160(8.4)$ & 1656 & $174(10.5)$ & $235(14.2)$ & $150(9.1)$ \\
\hline High School Grad or GED & 3162 & $299(9.5)$ & $387(12.2)$ & $241(7.6)$ & 3055 & $275(9.0)$ & $345(11.3)$ & $218(7.1)$ \\
\hline Some College & 2423 & $165(6.8)$ & $193(8.0)$ & $116(4.8)$ & 2595 & $218(8.4)$ & $208(8.0)$ & $133(5.1)$ \\
\hline Associates Degree & 877 & $37(4.2)$ & $43(4.9)$ & $18(2.1)$ & 989 & $54(5.5)$ & $58(5.9)$ & $40(4.0)$ \\
\hline Bachelors & 2921 & $114(3.9)$ & $135(4.6)$ & $58(2.0)$ & 2960 & $121(4.1)$ & $123(4.2)$ & $59(2.0)$ \\
\hline Master's Degree & 906 & $37(4.1)$ & $40(4.4)$ & $17(1.9)$ & 986 & $34(3.4)$ & $35(3.5)$ & $10(1.0)$ \\
\hline Doctorate or Professional & 241 & $12(5.0)$ & $10(4.1)$ & $5(2.1)$ & 242 & $12(5.0)$ & $11(4.5)$ & $8(3.3)$ \\
\hline \multicolumn{9}{|l|}{ Maternal Age (years) } \\
\hline 13-19 & 943 & $120(12.7)$ & $109(11.6)$ & $90(9.5)$ & 176 & $14(8.0)$ & $23(13.1)$ & $16(9.1)$ \\
\hline $20-24$ & 4086 & $354(8.7)$ & $485(11.9)$ & $276(6.8)$ & 2898 & $289(10.0)$ & 344 (11.9) & $222(7.7)$ \\
\hline $25-29$ & 4451 & $238(5.3)$ & $304(6.8)$ & $160(3.6)$ & 4132 & $314(7.6)$ & $363(8.8)$ & $220(5.3)$ \\
\hline $30-34$ & 2684 & $146(5.4)$ & $164(6.1)$ & $91(3.4)$ & 3956 & $206(5.2)$ & $247(6.2)$ & $128(3.2)$ \\
\hline $35-39$ & 648 & $34(5.2)$ & $40(6.2)$ & $23(3.5)$ & 1472 & $83(5.6)$ & $61(4.1)$ & $41(2.8)$ \\
\hline $40+$ & 65 & $3(4.6)$ & $3(4.6)$ & $1(1.5)$ & 243 & $14(5.8)$ & $18(7.4)$ & $12(4.9)$ \\
\hline \multicolumn{9}{|l|}{ WIC Recipient } \\
\hline Yes & 4930 & $463(9.4)$ & $574(11.6)$ & $362(7.3)$ & 4852 & $434(8.9)$ & $565(11.6)$ & $335(6.9)$ \\
\hline No & 7689 & $415(5.4)$ & $500(6.5)$ & $260(3.4)$ & 7946 & $478(6.0)$ & $488(6.1)$ & $297(3.7)$ \\
\hline
\end{tabular}

$\mathrm{SGA}=$ small for gestational age

LBW $=$ low birth weight

${ }^{a}$ Differences in race/ethnicity are noted between the second and third births. This can be attributed to differences in reporting by mothers at the time of each birth.

factors. Mixed study findings from the literature highlight the need for improved and consistent community education on IPI from both direct medical providers and community health workers [18].

\section{Limitations}

Data obtained from vital statistics is at risk for data entry errors, however, the state health department has methods in place to check the validity of hospital-entered data. The non-randomized methodology may limit the strength of study findings. Although the number of records included in analysis will helped address this limitation. The generalizability of this study is limited to developed countries.

\section{Conclusions}

Short IPIs may not be causally associated with low birthweight, preterm birth, and SGA but it remains a strong predictor of risk status among pregnant women $[14,19,20]$. Continued research is needed in this area to examine short IPIs with other birth outcomes and in other settings where nutritional deficiencies between pregnancies are more likely to occur.

Health care educators and health care providers should continue to use short birth spacing as an indicator of risk status and an opportunity for improved health education as part of a comprehensive strategy to improve birth outcomes and reduce health disparities. Additional training and resources are needed to further educate health educators on the shift in focus from a causal IPI and birth outcome relationship to one of risk identification.

\section{Acknowledgements}

The authors would like to thank Greg Crawford and Carol Moyer from the Kansas Department of Health and Environment for providing the vital statistic dataset and technical assistance. 
Table 2. Association between interpregnancy interval and adverse birth outcomes ${ }^{\mathrm{a}, \mathrm{b}}$.

\begin{tabular}{|c|c|c|c|c|}
\hline \multirow[t]{2}{*}{ IPI } & \multicolumn{2}{|c|}{ 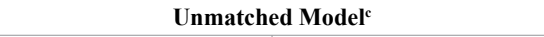 } & \multicolumn{2}{|c|}{ Matched Model } \\
\hline & Unadjusted & Adjusted $^{\mathrm{b}}$ & Unadjusted & Adjusted $^{\mathrm{b}}$ \\
\hline \multicolumn{5}{|c|}{ Preterm } \\
\hline $0-5$ & $2.54(2.04-3.16)$ & $1.98(1.58-2.47)$ & $1.46(1.13-1.88)$ & $1.42(1.10-1.84)$ \\
\hline 6-11 & $1.38(1.12-1.70)$ & $1.24(1.00-1.52)$ & $1.06(0.84-1.34)$ & $1.06(0.84-1.33)$ \\
\hline $12-17$ & $1.14(0.92-1.41)$ & $1.10(0.89-1.36)$ & $1.20(0.95-1.52)$ & $1.19(0.94-1.51)$ \\
\hline $18-23$ & 1.00 & 1.00 & 1.00 & 1.00 \\
\hline $24-59$ & $1.23(1.01-1.49)$ & $1.27(1.05-1.53)$ & $1.09(0.88-1.35)$ & $1.13(0.91-1.41)$ \\
\hline $60-119$ & $1.62(1.04-2.51)$ & $1.64(1.06-2.54)$ & $0.99(0.56-1.74)$ & $1.07(0.60-1.91)$ \\
\hline \multicolumn{5}{|c|}{ Small for Gestational Age } \\
\hline $0-5$ & $1.30(1.04-1.62)$ & $0.91(0.73-1.13)$ & $0.79(0.62-1.00)$ & $0.78(0.61-0.99)$ \\
\hline 6-11 & $1.06(0.87-1.28)$ & $0.90(0.74-1.08)$ & $0.88(0.72-1.08)$ & $0.88(0.71-1.08)$ \\
\hline $12-17$ & $1.01(0.83-1.22)$ & $0.98(0.81-1.18)$ & $0.96(0.78-1.18)$ & $0.95(0.77-1.17)$ \\
\hline $18-23$ & 1.00 & 1.00 & 1.00 & 1.00 \\
\hline $24-59$ & $1.01(0.85-1.20)$ & $1.07(0.90-1.26)$ & $1.03(0.85-1.24)$ & $1.04(0.86-1.26)$ \\
\hline $60-119$ & $1.17(0.76-1.80)$ & $1.20(0.79-1.84)$ & $0.80(0.49-1.33)$ & $0.86(0.52-1.44)$ \\
\hline \multicolumn{5}{|c|}{ Low Birth Weight } \\
\hline $0-5$ & $2.05(1.63-2.59)$ & $1.39(1.10-1.49)$ & $1.29(0.96-1.74)$ & $1.28(0.95-1.73)$ \\
\hline 6-11 & $1.12(0.90-1.40)$ & $0.93(0.75-1.16)$ & $0.87(0.66-1.14)$ & $0.87(0.66-1.14)$ \\
\hline $12-17$ & $0.91(0.73-1.15)$ & $0.87(0.70-1.09)$ & $1.01(0.76-1.33)$ & $1.00(0.76-1.32)$ \\
\hline $18-23$ & 1.00 & 1.00 & 1.00 & 1.00 \\
\hline $24-59$ & $1.09(0.89-1.33)$ & $1.16(0.95-1.41)$ & $1.06(0.82-1.37)$ & $1.08(0.84-1.40)$ \\
\hline 60-119 & $1.04(0.61-1.77)$ & $1.07(0.63-1.81)$ & $0.57(0.29-1.11)$ & $0.60(0.30-1.20)$ \\
\hline
\end{tabular}

${ }^{a}$ Values are presented as odds ratios $(95 \% \mathrm{CI})$ of preterm birth, small for gestational age, and low birth weight; 18-23 months is the reference for interpregnancy intervals.

${ }^{\mathrm{b}}$ Unmatched and matched models were adjusted for maternal age, parity, and use of WIC services

${ }^{\mathrm{c}}$ Missing data were noted in $1 \%$ of cases, therefore the unmatched analysis was limited to 12,754 births

\section{Funding}

This study was funded in-part by the Wichita Medical Research and Education Foundation Collaborative Investigator Initiated Research Funding Opportunity.

\section{References}

1. King JC (2003) The risk of maternal nutritional depletion and poor outcomes increases in early or closely spaced pregnancies. The Journal of nutrition 133: 1732S-1736S. [Crossref]

2. Conde-Agudelo A, Belizán JM, Norton MH, Rosas-Bermúdez A (2005) Effect of the interpregnancy interval on perinatal outcomes in Latin America. Obstetrics \& Gynecology 106: 359-366. [Crossref]

3. Auger N, Daniel M, Platt RW, Luo ZC, Wu Y, et al. (2008) The joint influence of marital status, interpregnancy interval, and neighborhood on small for gestational age birth: a retrospective cohort study. BMC Pregnancy Childbirth 8: 7. [Crossref]

4. DeFranco EA, Ehrlich S, Muglia LJ (2014) Influence of interpregnancy interval on birth timing. BJOG 121: 1633-1640. [Crossref]

5. Grisaru-Granovsky S, Gordon ES, Haklai Z, Samueloff A, Schimmel MM (2009) Effect of interpregnancy interval on adverse perinatal outcomes-a national study. Contraception 80: 512-518. [Crossref]

6. Hussaini KS, Ritenour D, Coonrod DV (2013) Interpregnancy intervals and the risk for infant mortality: a case control study of Arizona infants 2003-2007. Matern Child Health $J$ 17: 646-653. [Crossref]

7. Nerlander LM, Callaghan WM, Smith RA, Barfield WD (2015) Short interpregnancy interval associated with preterm birth in US adolescents. Matern Child Health $J$ 19:850-858. [Crossref]

8. Salihu HM, August EM, Mbah AK, Raymond J, Alio AP, et al. (2012) The impact of birth spacing on subsequent feto-infant outcomes among community enrollees of a federal healthy start project. Journal of community health 37: 137-142. [Crossref]

9. Crowne SS, Gonsalves K, Burrell L, McFarlane E, Duggan A (2012) Relationship between birth spacing, child maltreatment, and child behavior and development outcomes among at-risk families. Matern Child Health J 16: 1413-1420. [Crossref]

10. El-Kamary SS, Higman SM, Fuddy L, McFarlane E, Sia C, et al. (2004) Hawaii’s healthy start home visiting program: determinants and impact of rapid repeat birth. Pediatrics 114: e317-e326. [Crossref]

11. Teitler JO, Das D, Kruse L, Reichman NE (2012) Prenatal care and subsequent birth intervals. Perspect Sex Reprod Health 44: 13-21. [Crossref]

12. de Bocanegra HT, Chang R, Menz M, Howell M, Darney P (2013) Postpartum contraception in publicly-funded programs and interpregnancy intervals. Obstetrics \& Gynecology 122: 296-303. [Crossref]

13. de Bocanegra HT, Chang R, Howell M, Darney P (2014) Interpregnancy intervals: impact of postpartum contraceptive effectiveness and coverage. American journal of obstetrics and gynecology 210: 311. e311-311. e318. [Crossref]

14. Ball SJ, Pereira G, Jacoby P, de Klerk N, Stanley FJ (2014). Re-evaluation of link between interpregnancy interval and adverse birth outcomes: retrospective cohort study matching two intervals per mother. BMJ 349:g4333.

15. Kansas Department of Health and Environment (2014) Annual Summary of Vital Statistics, 2013.

16. Blumenshine P, Egerter S, Barclay CJ, Cubbin C, Braveman PA (2010) Socioeconomic disparities in adverse birth outcomes: a systematic review. Am J Prev Med 39: 263-272. [Crossref]

17. Lu M C, Lange L, Slusser W, Hamilton J, Halfon N (2001) Provider encouragement of breast-feeding: evidence from a national survey. Obstetrics \& Gynecology 97: 290295. [Crossref]

18. Schölmerich VL, Kawachi I (2016) Translating the Social-Ecological Perspective into Multilevel Interventions for Family Planning How Far Are We? Health Education \& Behavior 43: 246-255. [Crossref]

19. Conde-Agudelo A, Rosas-Bermúdez A, Kafury-Goeta AC (2006) Birth spacing and risk of adverse perinatal outcomes: a meta-analysis. JAMA 295: 1809-1823. [Crossref]

20. Conde-Agudelo A, Belizán, JM (2000) Maternal morbidity and mortality associated with interpregnancy interval: cross sectional study. BMJ 321: 1255-1259.

Copyright: (C2017 Woods NK. This is an open-access article distributed under the terms of the Creative Commons Attribution License, which permits unrestricted use, distribution, and reproduction in any medium, provided the original author and source are credited. 